\title{
Induced Infrared Thermography: Flow Visualization of Indoor Airflow over Heating surface
}

\author{
Qing $\mathrm{Wu}^{1,2}$, Jing Liu ${ }^{1,2^{*}}$, Chang-an Zhu ${ }^{3}$ \\ ${ }^{1}$ School of Architecture, Harbin Institute of technology, Harbin, China \\ ${ }^{2}$ Key Laboratory of Cold Region Urban and Rural Human Settlement Environment Science and \\ Technology, Ministry of Industry and Information Technology, Harbin Institute of Technology, \\ Harbin, China \\ ${ }^{3}$ China Southwest Architecture Design and Research Institute Co., Ltd, Chengdu, China
}

\begin{abstract}
The near-surface velocity can be measured by a method referred as "thermal image velocimetry", which acquires flow field according to the displacements of thermal spots in fixed intervals. Depending on the capability of twodimensional velocity visualization and the low system cost, this method is more advantageous over the other kinds of current velocity measurement methods. However, this method is still in the initial stage of development in indoor velocity measurement and feasibility of this method has not been fully verified. We aim to verify the feasibility of this method in the velocity measurement over the heating surface. Differ from the former studies, we conducted the experiments and explored the feasibility of this method in the condition of natural and mixed convection. The flow fields near the heat source were acquired by thermal image velocimetry. Particle image velocimetry was also used to compare the results evaluated by Thermal Image Velocimetry. The results show that the thermal image velocimetry can qualitatively and quantitatively reflect the near-surface velocity in all experimental conditions.
\end{abstract}

\section{Introduction}

In the current energy consumption system in China, building energy consumption accounts for a large proportion (Prek and Krese, 2018). In addition, in the energy consumption of buildings, the energy consumption for heating should not to be underestimated. With the gradual increase of energy consumption, the depletion of fossil energy and the deterioration of the environment have become more and more serious. Consequently, much attention have been focused on improving the efficiency of heating system. On the other hand, it is necessary to ensure indoor thermal comfort while implementing measures such as reducing the heating temperature to decrease the heating energy consumption.

In China, radiators are still the most widely used heating terminal equipment (Luo and Wang, 2018). The reasonable arrangement of the radiator is of great significance for lifting the energy efficiency of the heating system and improving the indoor thermal environment. As heat source in room, radiator transmits energy to the nearby air mainly through the form of convection. The heated air rises along vertical direction. As the hot air rises, the insufficient driving force of the thermal buoyancy causes the hot air to escape from the original flow direction. On the other hand, the cold air far away from the radiator in the room will continuously flow to the vicinity of the radiator to replenish, thus making the air circulate throughout the room. Indoor air is thus heated, offsetting the discomfort caused by the heat loss of the maintenance structure or the outdoor cold air penetration.

As the indoor hot air rises and the cold air sinks, a relatively stable temperature and airflow distribution is finally established. Therefore, the flow state of the hot air over radiator largely determines the airflow organization and energy efficiency of the entire room (Awbi, 1998; Cholewa and Anasiewicz, 2017; Peeters and BeausoleilMorrison, 2011). Accurately obtaining the flow of airflow over the radiator is very important for evaluating indoor airflow distribution and indoor heating design. For the time being, there are mainly two kinds of indoor velocity measurements, which are point-wise measurement and global-wise measurement (Sun and Zhang, 2007). The global-wise measurement method such as particle image velocimetry can visualize the two or three dimensional airflow field and is helpful to understand the flow characteristics (Gao and Shaw, 1989). The limitations of these two velocity measurement methods for the nearsurface flow field over the heat source has been debated ( $\mathrm{Wu}$ and Liu, 2019). The interaction of near surface turbulent structures and the surface temperature confirmed to be closely related (Castellví and Cammalleri, 2016; Aminzadeh and Breitenstein, 2017), which brings a new perspective for the measurement of near-surface flow. The high frequency temperature fluctuations can be obtained by infrared thermography. Several studies have been carried out to obtain near-surface flow field by measuring the high frequency surface temperature fluctuations. However, most of the researches were conducted outdoors and are rarely applied in indoor environment, especially the flow field above vertical heat source. In the current research, there are two main methods to deduce the flow conditions by infrared thermography. One is to directly establish the relationship between near-surface velocity and temperature through theoretical analysis. The other is similar to the particle image velocimetry technology and obtains the nearsurface flow field by analysing the displacements of temperature spots between continuous frames.

The method based on surface-renewal theory

When turbulent structures flow through surface, the interaction between the turbulent flow and surface affects 
the energy exchange (Paw and Qiu, 1995). As the airflow flows through the surface, the energy exchange is promoted or inhibited. There is a direct relationship between the flow near the wall and the wall temperature fluctuations consequently. The energy transfer contributed by the turbulent structures can be quantified by the surface renewal theory. In this theory, the interaction process between airflow and surface is explained as the intermittent residence of flow eddies on the surface (Haghighi and Or, 2013). When the flow eddies stay on the surface, thermal exchange occurs between the turbulent structures and the surface, and the surface temperature pattern changes correspondingly. The near-surface airflow is described as a collection of the flow eddies with different sizes and intensities (Paw and Qiu, 1995). The intermittent residence time of the flow eddies can be described by the eddy spectrum shape and rate parameters and is distributed in gamma function. The thermal exchange between the surface and turbulent eddies mainly occurs in the viscous sublayer, whose thickness characterizes the thermal exchange of the flow eddies and surface. Haghighi and Or (2013) proposed a function which connects the near-surface velocity with the thickness of viscous sublayer:

$$
\delta=\frac{\alpha f(\alpha) v_{a}}{x \bar{u}}
$$

where $\delta$ is the thickness of the viscous sublayer, $\alpha$ is the eddy spectrum shape parameter deduced from the surface temperature fluctuations, $v_{a}$ is the kinematic viscosity of air, $x$ is a dimensionless parameter which is determined by the flow characteristic and surface properties, $\bar{u}$ is the mean flow velocity near the surface.

The surface heat exchange is composed of two parts, which are the radiative and sensible heat fluxes, when no latent heat fluxes exists. The sensible heat exchange occurs within the viscous sublayer.

$$
H=\left.k_{a} \frac{\partial T}{\partial z}\right|_{z=0}=\frac{k_{a}}{\delta} \Delta T
$$

where $H$ is the sensible heat flux, $k_{a}$ is the thermal conductivity of the air, $\Delta T$ is the temperature difference of the air and surface. Combing (1) and (2), the relationship between the surface temperature fluctuation and near-surface mean flow velocity can be established. Based on this method, the near-surface mean flow velocity can be obtained.

\section{Thermal image velocimetry}

Thermal image velocimetry (TIV) is similar to the particle image velocimetry, which estimates the surface twodimensional velocity distributions. As mentioned earlier, when airflow flows through the surface, the flow can induce the surface temperature fluctuations. In the method of thermal image velocimetry, the flow condition is inversely deduced by the temperature fluctuations. The high frequency temperature fluctuations are obtained by the infrared thermography. As a result, this method can also be referred as 'induced infrared thermography'. A sequence of image proceeding steps were performed to visualize the near-surface velocity according to sequences of thermal images. The whole data processing are as follows: (1) Preparing thermal images. In this step, the high-frequency surface temperature fluctuations are captured using infrared camera and saved in the form of sequences of infrared thermal images. (2) Image Preprocessing. This step aims at accentuating the movements of thermal spots compared to the background noise. A high-pass filter is used to reduce the influence of the background noise. (3) Image processing. In this step, the velocity vectors are calculated using the calculating process similar to particle image velocimetry (PIV). The thermal spot is similar to the tracer particle in PIV, and the displacements of the thermal spots are obtained by cross-correlation calculation within the interrogation window. With the shift of the interrogation window, the entire flow filed of the image is visualized. (4) Data correction. In the raw two-dimensional velocity flow field obtained in the previous step, there are some error vectors and need to be removed. The error vectors are tracked and removed in this step. More detailed data processing can be referred in $\mathrm{Wu}$ and Liu (2019).

Compared to the method based on the surface renewal theory, this method can visualize the near-surface twodimensional velocity field. Restricted to the geometrical constrains and the magnitude of velocity, the vertical velocity gradient of the airflow field above the heat source is large. The point-wise velocity information cannot reflect the vertical distribution characteristics. In consequence, thermal image velocimetry method is more suitable for the measurement of near-surface flow field above the heat source. Thermal image velocimetry method has been used in some outdoor studies (Huang and Hwang, 2015; Inagaki and Kanda, 2013; Legleiter and Kinzel, 2017; Lopez and Thomas, 2014; Tiddens and Risthaus, 2017; Lev and Spiegelman, 2012), but rarely used in indoor study, especially in the visualization of the flow field above the indoor heat source.

In our former study (Wu and Liu, 2019), the feasibility of thermal image velocimetry in the velocity measurement over the heat source under natural condition has been verified. Differ from the former study, this study aims at exploring the feasibility of thermal image velocimetry both in the condition of natural convection and mixed convection. In addition, the influence of heat source surface temperature and ventilation on the near-wall flow over the heat source was also measured and analysed.

\section{Method}

Experiments were performed in a cavity, of which the internal dimension is $1200 \times 500 \times 1400 \mathrm{~mm}^{3}$. The walls are made of Plexi-glass and the surface emissivity is 0.90 . A heating film with the dimension of $500 \times 400 \mathrm{~mm}^{2}$ is used as the heat source in the cavity. A $20 \mathrm{~mm}$ wide ventilation inlet is installed at the bottom of the cavity near the heated vertical wall. The cavity is connected with fan and thus forming a closed circulation system. The experimental cavity is shown as Figure 1. 


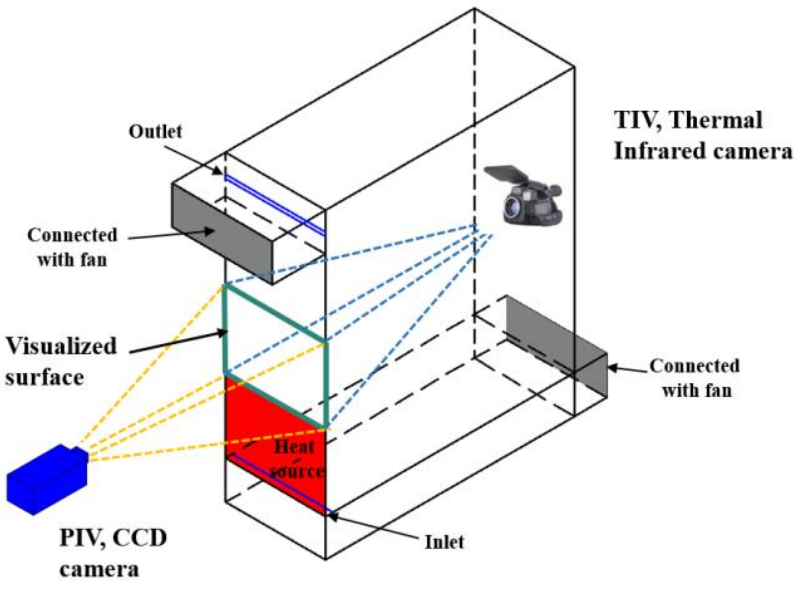

Figure 1: Schematic view of the experimental setup.

The surface temperature fluctuations of the wall over the heat source was obtained by a FLIR-T1040 thermal infrared camera. For comparison with TIV measurements, PIV was used to measure the velocity distribution of the plane that is $8 \mathrm{~mm}$ from the heated vertical wall. The visualized surface of PIV and TIV is $500 \times 400 \mathrm{~mm}^{2}$, shown as Figure 1. Infrared camera and the CCD camera of PIV captured the target flow field from opposite sides. Infrared thermal camera obtained the surface temperature fluctuations from the shooting positions located in $600 \mathrm{~mm}$ away from the bottom of the cavity. The relevant parameters of PIV system are shown as Table 1.

\section{Table 1: PIV parameters.}

\begin{tabular}{|c|c|}
\hline & Parameters \\
\hline Laser & Double-pulsed Nd:YAG \\
\hline CCD & PIVCAM13-8 \\
\hline Lens & AF Nikon 50mm lens \\
\hline Laser intensity & $150 \mathrm{~mJ} /$ pulse \\
\hline Visual field & 2048 pixel $\times 2048$ pixel \\
\hline Smoke generator & VZ09-0751 \\
\hline Sampling frequency & $7 \mathrm{~Hz}$ \\
\hline Images & 600 \\
\hline Interrogation window & 32 pixel $\times 32$ pixel \\
\hline Overlap & $50 \%$ \\
\hline
\end{tabular}

The parameters of infrared camera are shown as Table 2. The laser intensity of PIV system is $150 \mathrm{~mJ}$ and the sensitive band range of thermal infrared camera is 7.5$14 \mu \mathrm{m}$.

Table 2: Infrared camera parameters.

\begin{tabular}{|c|c|}
\hline & Parameters \\
\hline Model & FLIR T1040 \\
\hline Resolution & 1024 pixel $\times 768$ pixel \\
\hline Sensitive wave & $7.5 \sim 14 \mu \mathrm{m}$ \\
\hline Angle of version & $28^{\circ} \times 21^{\circ}$ \\
\hline Sampling frequency & $30 \mathrm{~Hz}$ \\
\hline Images & 600 \\
\hline
\end{tabular}

The experiments were divided into three cases. The experimental cases were adjusted by changing the surface temperature of the heat source and the ventilation velocity. Case 1 is under natural convection, and the surface temperature of heat source is $45^{\circ} \mathrm{C}$. Case 2 is under natural convection, and the surface temperature of heat source is $65^{\circ} \mathrm{C}$. Case 3 is under mixed convection, and the surface temperature is $65^{\circ} \mathrm{C}$. The velocity of the ventilation inlet is $1.75 \mathrm{~m} / \mathrm{s}$.

\section{Results}

The instantaneous velocities are averaged and the timeaveraged velocity counters and vectors of the three cases are shown as Figure 2 to Figure 7.

Figure 2 and Figure 3 are the velocity distributions and vectors of Case 1 measured by PIV and TIV. The velocity distribution exhibits spatial difference in the vertical direction, which is agree with the analysis the introduction section. As can be seen from the figures, the region where the maximum velocity appears is in the vertical range of $200 \mathrm{~mm}-400 \mathrm{~mm}$. Overall, the velocity increases with the increase of the vertical position.

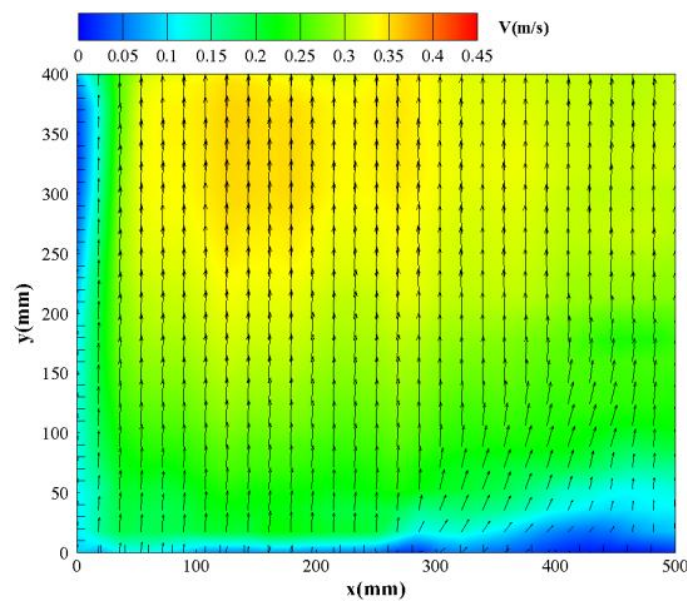

Figure 2: Velocity distribution of Case1 measured by PIV.

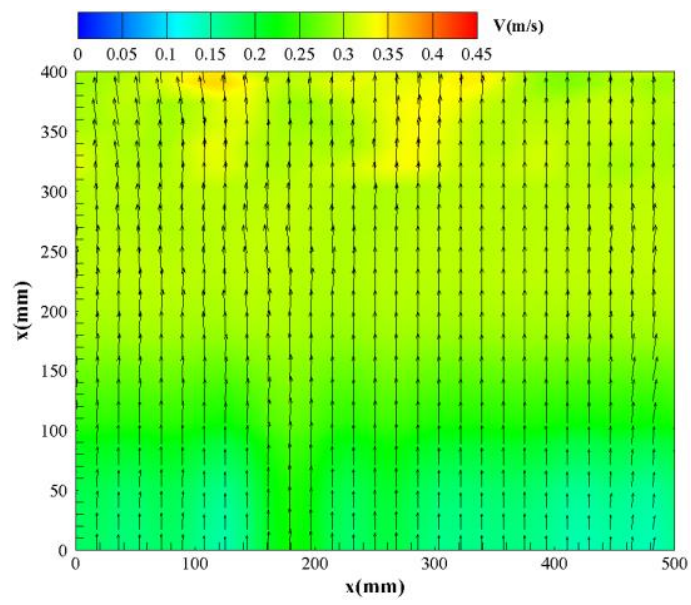

Figure 3: Velocity distribution of Case1 measured by $T I V$.

Figure 4 and Figure 5 are the velocity distributions and vectors of Case 2 measured by PIV and TIV. The velocity distribution exhibits more pronounced spatial difference in the vertical direction, compared with the results of Case1. As can be seen from the figures, the region where the maximum velocity appear is in the vertical range of $350 \mathrm{~mm}-400 \mathrm{~mm}$. With the surface temperature of the heat source, the position at which the maximum velocity occurs moves up. In addition, the magnitude the maximum velocity increases with the increase of the surface temperature of the heat source. 


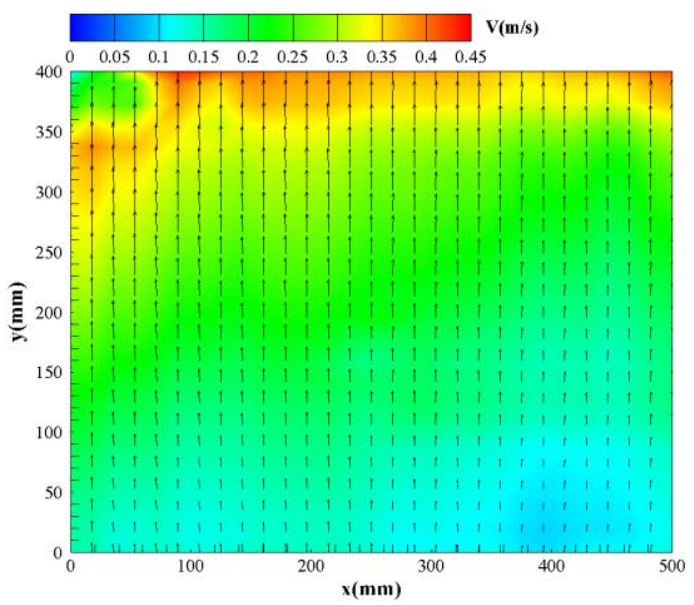

Figure 4: Velocity distribution of Case 2 measured by PIV.

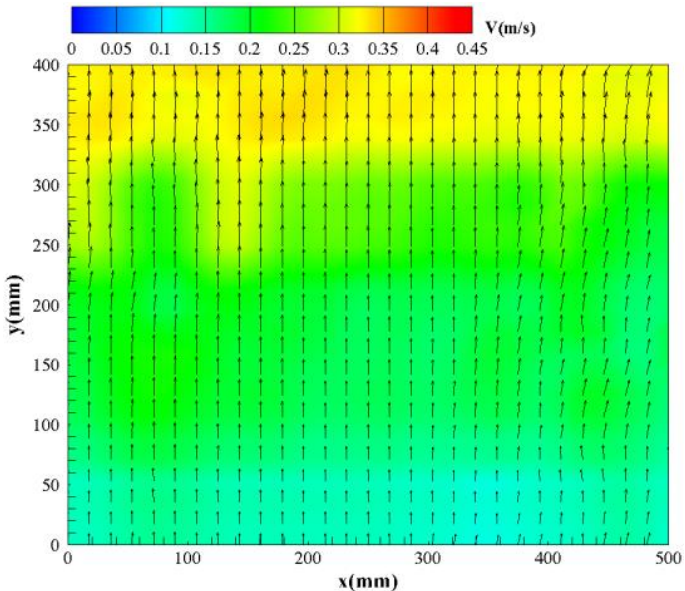

Figure 5: Velocity distribution of Case2 measured by TIV

Figure 6 and Figure 7 are the velocity distributions and vectors of Case 3 measured by PIV and TIV. The velocity distribution exhibits a certain spatial difference in the vertical direction. Differ from Case 1 and Case 2, Case 3 is under the condition of mixed convection. The natural convection and mechanical ventilation make a joint effect on the velocity distribution. As can be seen from the figures, the region where the maximum velocity appears is in the vertical range of $0 \mathrm{~mm}-200 \mathrm{~mm}$, which is the location close to the ventilation inlet. The overall distribution of velocity shows a decreasing trend with the increase of vertical position. The velocity distribution is changed under the effect of mechanical ventilation, indicating that the natural convection effect is weaker than the effect of mechanical ventilation.

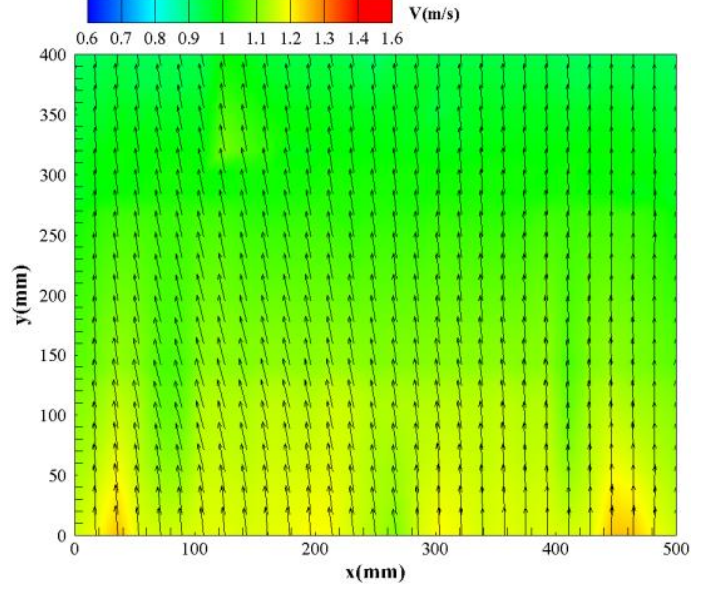

Figure 6: Velocity distribution of Case2 measured by PIV.

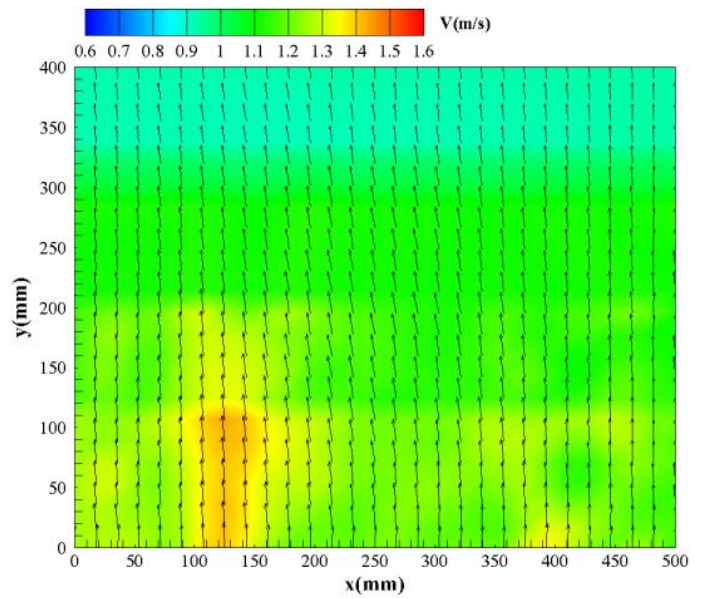

Figure 7: Velocity distribution of Case 2 measured by $T I V$.

\section{Discussion}

From the perspective of velocity distribution, TIV measurement results are close to the PIV measurement results. The specific positions of the three cases where the maximum velocity occur are close to the PIV, indicating that the TIV measurement results can reflect the spatial distribution characteristics of the velocity.

From the perspective of the magnitude of maximum velocity, the measurement results of TIV are slightly smaller than the measurement results of PIV. In addition to conditions such as the resolution of the camera, differences in the principles of the measurement methods may cause this difference. Since the TIV measurement method indirectly obtains the near-surface flow velocity by measuring the temperature fluctuations of the wall surface, the wall temperature conditions directly affect the measurement result. With the change of surface temperature of heat source and the ventilation velocity, the thickness of the boundary layer near the visualized wall changes accordingly. Therefore, the flow field position measured by TIV changes, but the position of the PIV is fixed at $8 \mathrm{~mm}$. For different working conditions, the position of flow field measured by TIV is not fixed, but it is certain that the result obtained by TIV is associated with the thickness of near-wall boundary layer. 
It is worth noticing that the verification of feasibility of thermal image velocimetry was achieved in lad condition rather than field measurement. Scaled model experimental research still has a certain difference with the actual condition and the difference is mainly caused by the physical parameters of the heat source. The shape of the actual radiator is not a plane, and the size of the heat source relative to the entire room are inconsistent with the scaled model experiment. The shape of the actual radiator surface is more complicated, and the flow above the heat source in the actual room may be different from that in the scaled model experiment. In actual room, the vertical surface over the heat source is not always homogeneous like the scaled model experiment. There may be some materials such as glass that are different from the wall material, and thus the surface temperature fluctuations are inconsistent. This will add some difficult to the measurement of airflow field using thermal image velocimetry method.

However, through setting the corresponding time filter, the influence of the background heterogeneity can be overcame (Inagaki and Kanda, 2013; Gonzalez and Woods, 2003). Although the physical parameters of the heat surfaces are not identical, the airflow field measured in this study is the same as the flow over the actual radiator and are both the flow above the vertical heat surface. Based on the measurement results of thermal image velocimetry method under natural and mixed convection condition, we believe that this method has great potential in the measurement of airflow field above indoor heat source.

Due to the difficulty of airflow field measurement near the heat source, most studies on indoor airflow are focused on the airflow situation in the work region. As stated in the introduction section, the airflow near the heat source plays an important role in the airflow distribution in the entire room. Due to the lack of the flow field measurement information near the heat source, most of the numerical studies verify the correctness of the simulation results by comparing the simulation results of the working area with the measured values. Therefore, no more accurate determination can be made on the simulation results near the heat source. Thermal image velocimetry method is a non-intrusive and the twodimensional velocity distributions can be captured. If this method is applied in the measurement of airflow field near the indoor heat source, the simulation results near the heat source will be netter verified, and the it is also more conductive to the study of the overall distribution of the indoor airflow field.

PIV is a mature flow field visualization method, and there are some factors that influence the measurement accuracy, which is also the main source of errors in this study. The uncertainty of PIV measurement consists of two parts, which are the system error and the statistical error. The performance of tracing particles, image deformation, and random displacement are the main factors in system errors (Cao, 2015). The maximum system error is about $4.3 \%$ (Cao, 2015; Raffel and Willert, 2007). The statistical error can be evaluated by estimating the random sampling error.
The random sampling error of the time-averaged velocity can be evaluated as (Coleman and Steel, 2009; Stafford and Walsh, 2012):

$$
S(A)=\frac{1}{\sqrt{k}} \cdot z_{\alpha / 2} \cdot \frac{a^{\prime}}{A}
$$

where $S(A)$ is the random sampling error, $k$ is the sampling size, $a^{\prime}$ is the root mean square velocity, $A$ is the timeaveraged velocity, $z_{\alpha / 2}$ is determined by coefficient interval and is equal to 1.96 when the confidence interval is $95 \%$. The calculated random sampling error of three cases are shown in Figure 8 . The random sampling error of the three cases are about $0.35 \%$ and can be neglected. Therefore, the uncertainty of the PIV measurement is about $4.4 \%$.

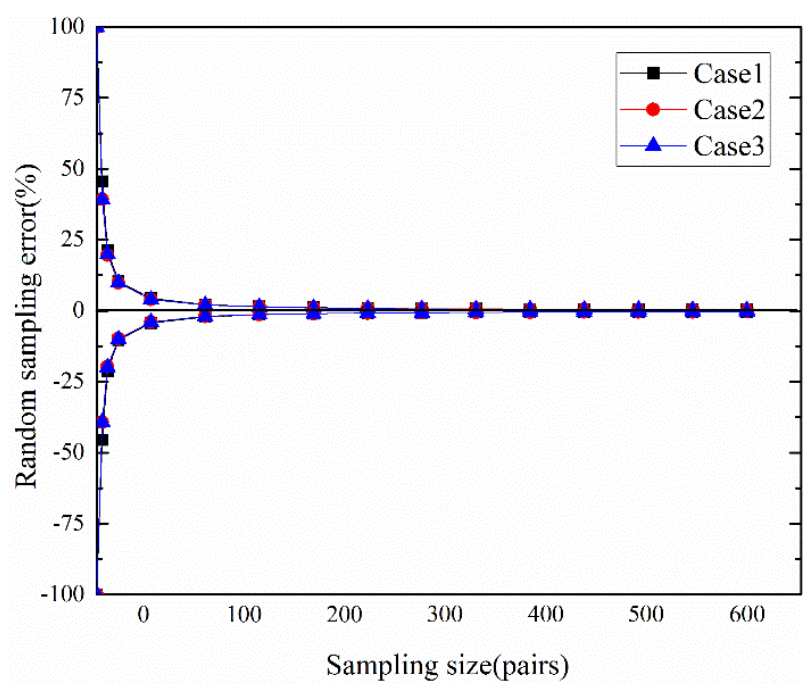

Figure 8: Random sampling error.

\section{Conclusion}

Thermal image velocity measurement is a velocity measurement method that obtains the velocity distributions based on the high frequency temperature fluctuations. Compared to the other kinds of current velocity measurement methods, this method is more advantageous due to its ability of flow visualization and low system cost.

This study compared the results of thermal image velocimetry both in the condition of natural convection and mixed convection. From the perspective of velocity distribution, the measurement results of thermal image velocimetry are close to the measurement results of particle image velocimetry. The results obtained by the two methods have similar distribution trend and magnitude.

Due to the interaction between the air and surface, the thickness of the near-surface boundary layer affects the fluctuations of the surface temperature. In thermal image velocimetry method, the near-surface velocity is obtained indirectly by detecting the surface temperature fluctuations. Therefore, the surface temperature conditions directly affect the measurement result.

In addition, the influence of surface temperature of the heat source and ventilation velocity on the near-wall flow 
over the heat source was analysed. In natural convection, the airflow velocity in the top region is lager driven by thermal buoyancy. In addition, the vertical distribution gradient is more obvious, as the surface temperature of the heat source increases. After mechanical ventilation, the original trend of velocity distribution is broken. The velocity tends to increase as closer to the ventilation inlet located in the bottom of the cavity.

\section{Acknowledgement}

This study was supported by "the 13th Five-Year" National Key R\&D Program of China (Grant No. 2017YFC0702200).

\section{References}

Aminzadeh, M. and D. Breitenstein (2017). Characteristics of Turbulent Airflow Deduced from Rapid Surface Thermal Fluctuations: An Infrared Surface Anemometer. Boundary-Layer Meteorol 165(6), 1-16.

Awbi, H.B. (1998). Calculation of convective heat transfer coefficients of room surfaces for natural convection. Energy Build. 28, 219-227.

Castellví, F. and C. Cammalleri (2016). Daytime sensible heat flux estimation over heterogeneous surfaces using multitemporal land-surface temperature observations. Water Resource Research 52(5), 34573476.

Cao, X.D. (2015). Experimental study of the airflow characteristics in a passenger aircraft cabin mockup with 2D-PIV. Tianjing university.

Cholewa, T. and R. Anasiewicz (2017). On the heat transfer coefficients between heated/cooled radiant ceiling and room. Applied Thermal Engineering 117, 76-84.

Coleman, H.W., Steel, W.G. (2009). Experimental, validation, and uncertainty analysis for engineers. John wiley \&Sons. New jersey (USA).

Gao, W. and R.H. Shaw (1989). Observation of organized structure in turbulent flow within and above a forest canopy. Boundary-Layer Meteorol 47, 349-377

Gonzalez, R. C., Woods, R. E. (2003). Digital Image processing. Prentice Hall. New jersey (USA).

Haghighi, E. and D. Or (2013). Evaporation from porous surfaces into turbulent airflows: Coupling eddy characteristics with pore scale vapor diffusion. Water Resource Research 49, 8432-8442.

Huang, Z.C. and K.S. Hwang (2015). Measurements of surface thermal structure, kinematics, and turbulence of a large-scale solitary breaking wave using infrared imaging techniques. Coast. Eng. 96, 132-147.
Inagaki, A. and M. Kanda (2013). Thermal Image Velocimetry. Boundary-Layer Meteorol 149, 1-18.

Legleiter, C.J. and P.J. Kinzel (2017). Remote measurement of river discharge using thermal particle image velocimetry (PIV) and various sources of bathymetric information. J. Hydrol. 554, 490-506.

Lev, E. and M. Spiegelman (2012). Investigating lava flow rheology using video analysis and numerical flow models. J. Volcanol. Geotherm. Res 248-248, $62-73$.

Lopez, T. and H.E. Thomas (2014). Volcanic plume characteristics determined using an infrared imaging camera. J. Volcanol. Geotherm. Res. 300, 148-166.

Luo, M. and Z. Wang (2018). Indoor climate experience, migration, and thermal comfort expectation in buildings. Bulding and environment 141, 262-272.

Paw, U.K.T. and J. Qiu (1995). Surface renewal analysis: a new method to obtain scalar fluxes. Agric. For. Meteorol 74, 119-137.

Peeters, L. and I. Beausoleil-Morrison (2011). Internal convective heat transfer modeling: Critical review and discussion of experimentally derived correlations. Energy Build. 43, 2227-2239.

Prek, M. and G. Krese (2018). Experimental analysis of an improved regulation concept for multi- panel heating radiators: Proof-of-concep. Energy 161, 5259.

Raffel, M., Willert, C., (2007). Particle image velocimetry: a paractical guide, $2^{\text {nd }}$ edition. Springer. Berlin (Germany).

Stafford, J. and E.Walsh (2012). A statistical analysis for time-averaged turbulent and fluctuatiing flow field using particle image velocimetry. Flow Means. Instrum. 26, 1-9.

Sun, Y. and Y. Zhang ( 2007). An overview of room air motion measurement: technology and application. $H V A C \& R \operatorname{Res} 13(6), 929-950$.

Tiddens, A. and K. Risthaus (2017). Induced Infrared Thermography: Flow visualizations under the extreme conditions of an open volumetric receiver of a solar tower. Int. J. Heat Fluid Flow 65, 105-113.

$\mathrm{Wu}$, Q. and J. Liu (2019). Two-dimensional flow visualization and velocity measurement in natural convection near indoor heated surfaces using a thermal image velocimetry method. Applied thermal engineering 146, 556-568. 